\title{
The rapidly pulsating subdwarf B star PG 1325+101
}

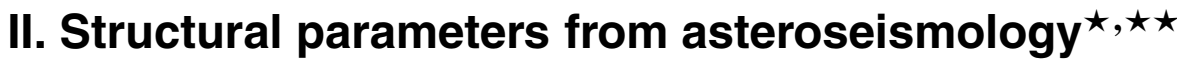

\author{
S. Charpinet ${ }^{1}$, R. Silvotti ${ }^{2}$, A. Bonanno ${ }^{3}$, G. Fontaine ${ }^{4}$, P. Brassard ${ }^{4}$, P. Chayer ${ }^{5,6}$, E. M. Green ${ }^{7}$, P. Bergeron ${ }^{4}$, \\ S. Bernabei ${ }^{8}$, S. Leccia ${ }^{3}$, H. Kjeldsen ${ }^{9}$, R. Janulis ${ }^{10}$, A. Frasca ${ }^{3}$, R. Østensen ${ }^{11}$, S.-L. Kim ${ }^{12}$, B.-G. Park ${ }^{12}$, X. Jiang ${ }^{13}$, \\ M. D. Reed ${ }^{14}$, R. S. Patterson ${ }^{14}$, K. M. Gietzen ${ }^{14}$, P. J. Clark ${ }^{14}$, G. W. Wolf ${ }^{14}$, Y. Lipkin ${ }^{15}$, L. Formiggini ${ }^{15}$, \\ E. Leibowitz ${ }^{15}$, T. D. Oswalt ${ }^{16}$, M. Rudkin ${ }^{16}$, and K. Johnston ${ }^{16}$
}

(Affiliations can be found after the references)

Received 29 March 2006 / Accepted 11 May 2006

\begin{abstract}
We present a detailed analysis of the rapidly pulsating subdwarf B star (or EC14026 star) PG 1325+101. This analysis exploits the outcome of dedicated multisite observations using time-series photometry that revealed the presence of (at least) 15 periodicities in the luminosity modulation of this star (Silvotti et al. 2006, A\&A, 459, 557, Paper I). Based on high- $S / N$, medium-resolution spectra obtained at the Steward Observatory's $2.3 \mathrm{~m}$ Telescope and at the $6.5 \mathrm{~m}$ MMT Telescope, new NLTE spectroscopic derivations of the atmospheric parameters of PG $1325+101$ are presented. The mean values of $T_{\text {eff }}=35050 \pm 220 \mathrm{~K}, \log g=5.81 \pm 0.04$, and $\log N(\mathrm{He}) / N(\mathrm{H})=-1.70 \pm 0.02$ are in agreement with previous determinations, but are significantly more accurate. These improved spectroscopic parameters are essential for isolating a unique asteroseismic model solution for the observed pulsation spectrum of PG 1325+101. Using the "forward modeling" approach, our combined spectroscopic and asteroseismic analysis leads objectively to the identification of the $(k, \ell)$ indices of the 12 independent modes observed in this star, and to the determination of its structural parameters. The periods correspond to low-order acoustic modes with adjacent values of $k$ and having degrees $\ell=0-4$. They define a band of unstable modes, in agreement with nonadiabatic pulsation theory. The average dispersion achieved between the observed periods and the periods of the corresponding theoretical modes of the optimal model is only $\sim 0.46 \%$ ( $\sim 0.54 \mathrm{~s})$, comparable to the results from similar analyses of other EC14026 stars analysed to date. The inferred structural parameters of PG $1325+101$ are $T_{\text {eff }}=35050 \pm 220 \mathrm{~K}$, $\log g=5.811 \pm 0.004, \log M_{\text {env }} / M_{*}=-4.18 \pm 0.10, M_{*}=0.50 \pm 0.01 M_{\odot}$ (i.e., close to the canonical mass of extreme horizontal branch stars), $R / R_{\odot}=0.145 \pm 0.002$, and $L / L_{\odot}=28.3 \pm 1.5$. In addition, by combining detailed model atmosphere calculations with $V=14.019 \pm 0.012$, we estimate that this star has an absolute visual magnitude $M_{\mathrm{V}}=4.45 \pm 0.04$ and is located at a distance of $d=820 \pm 21$ pc. Finally, the presence of fine structure in the observed period spectrum suggests a rotation period of $P=1.6 \pm 0.2$ days, leading to an equatorial velocity of $V_{\mathrm{eq}}=4.6 \pm 0.6 \mathrm{~km} \mathrm{~s}{ }^{-1}$. Hence, asteroseismic evidence suggests that PG $1325+101$ is a slow rotator, a conclusion reinforced by the limit $V \sin i<20-30 \mathrm{~km} \mathrm{~s}{ }^{-1}$, which we determined by modeling the He I 4471 line in our $1 \AA$-resolution MMT spectrum.
\end{abstract}

Key words. stars: interiors - stars: oscillations - stars: horizontal-branch - stars: individual: PG $1325+101$

\section{Introduction}

Probing the inner layers of stars using the pulsations they might exhibit - a field that we refer to as asteroseismology - has been a goal actively pursued for more than two decades. Indeed, oscillating stars allow a direct investigation of their structure from the analysis of pulsation modes, the eigenfrequencies of a selfgravitating gaseous sphere. These frequencies (or periods) are determined by internal properties which are otherwise difficult or impossible to measure directly. Considering that our current knowledge of stars relies almost exclusively on what is seen at their surfaces - the properties of their hidden, inner layers being deduced only from modeling - the promise of extracting direct information on their internal structure through asteroseismology has tremendous implications.

Among all known classes of pulsating stars spanning the HRDiagram, the group of rapidly pulsating hot subdwarf B (sdB)

* Some spectroscopic observations reported here were obtained at the MMT Observatory, a joint facility of the University of Arizona and the Smithsonian Institution.

$\star \star$ This study made extensive use of the computing facilities offered by the Calcul en Midi-Pyrénées (CALMIP) project, France. stars (or EC14026 stars) has so far provided excellent opportunities for pursuing asteroseismic studies. Subdwarf B stars dominate the populations of faint blue stars down to $V \sim 16$ and are found in both the old disk (field sdBs) and halo populations (globular cluster members) of our own Galaxy. They are the most likely source of the UV excess observed in elliptical galaxies (Brown et al. 1997) and have been identified (Heber 1986; Saffer et al. 1994) with models of Extreme Horizontal Branch (EHB) stars burning He in their cores and having extremely thin residual H-rich envelopes (Dorman et al. 1993). Significant holes persist in our understanding of this phase of stellar evolution, however. For instance, the mechanisms that lead to their formation are still being debated. The main contenders are single star evolution with enhanced mass loss at the tip of the red giant branch and various scenarios involving binary evolution (see Han et al. 2003, and references therein). Furthermore, there remain important details concerning the exact behavior of the helium burning cores during this stage of evolution that are not yet understood. So far, classical observation and modeling techniques have been unable to provide answers to these basic questions, and thus new approaches to constrain the global structure of EHB stars are needed. 
Asteroseismology has become a valuable technique now that a significant number of $\mathrm{sdB}$ stars have been discovered to be pulsators, similar to the one first identified a few years ago by Kilkenny et al. (1997). About three dozen EC14026 variables, with $T_{\text {eff }} \sim 30000-36000 \mathrm{~K}$ and periods in the range 100-600 s, are now known. Their pulsations are associated with low-order low-degree $p$-modes and are driven by an opacity peak in the $\mathrm{sdB}$ envelope which is mainly due to iron locally enhanced by radiative levitation (Charpinet et al. 1997, 2001). More recently, Green et al. (2003) established that some of the cooler B subdwarfs may belong to a second, seemingly independent class of multiperiodic pulsators with significantly longer periods. These long period sdB variables (the PG $1716+426$ pulsators, sometimes also referred to as the "Betsy" or the "lpsdBV" stars) populate the low-temperature/low-gravity corner $\left(T_{\text {eff }} \lesssim 30000 \mathrm{~K}\right.$, $\log g \lesssim 5.7)$ of the region in the $\log g-T_{\text {eff }}$ plane where $\mathrm{sdB}$ stars are found. Their multiperiodic luminosity variations occur on timescales of $\sim 0.75-2.0 \mathrm{~h}$ (i.e., much longer than the periods observed in the EC14026 stars), implying that relatively highorder gravity modes are involved. The same mechanism responsible for the oscillations in the EC14026 stars has been proposed to operate in the long-period sdB pulsators as well, in this case destabilizing high-order, $\ell \gtrsim 3$ gravity modes (Fontaine et al. 2003).

While it is too early to assess the real asteroseismological potential of the newly discovered PG 1716+426 stars (but see Randall et al. 2006), the EC14026 pulsators have proven to be highly suitable for detailed asteroseismic studies. This has been demonstrated for the pulsating sdB stars PG 0014+067 (in the pioneering work of Brassard et al. 2001), PG1047+003 (Charpinet et al. 2003), PG 1219+534 (Charpinet et al. 2005b) and Feige 48 (Charpinet et al. 2005a), all based on high $S / N$ "white light" photometry gathered at the Canada-France-Hawaii Telescope. Close simultaneous matches of all periodicities, as well as the determination of fundamental structural parameters of these stars were achieved using a new global optimization technique. In every case, mode identifications (i.e., the determination of the radial order $k$ and degree $\ell$ ) for all of the observed modes have also been derived.

Continuing the asteroseismic studies mentioned above, we now present the results of a detailed analysis of the rapidly pulsating sdB star PG $1325+101$. The variability of this star was first announced by Silvotti et al. (2002) during a survey conducted at the Nordic Optical Telescope (Solheim et al. 2004). This discovery was followed by a significant observational effort to obtain high frequency resolution seismic data for this star. In Paper I (Silvotti et al. 2006), we presented the results of about 215 hours of time-series photometry acquired for PG $1325+101$ at nine different sites during 25 nights of observation in the spring of 2003. The resulting analysis clearly reveals the presence of 15 periodicities, with possible additional signal still remaining in the residual light curves. The rather rich pulsational spectrum obtained for PG $1325+101$ has thus revealed the high potential of this star for asteroseismic probing. In Sect. 2, we propose new improved estimates of the atmospheric parameters of PG $1325+101$ obtained from high- $S / N$, medium-resolution spectra. These will prove essential in the course of the detailed asteroseismic analysis described in Sect. 3. Major conclusions resulting from this analysis are then summarized in Sect. 4.

\section{Atmospheric parameters from spectroscopy}

The atmospheric parameters of PG $1325+101$ were first estimated by Saffer et al. (1994) who gave $T_{\text {eff }}=34500 \pm 1000 \mathrm{~K}$, $\log g=6.11 \pm 0.15$, and $\log N(\mathrm{He}) / N(\mathrm{H})=-1.68 \pm 0.17$ on the basis of a rather low $S / N$ ratio $(\sim 20)$ optical spectrum. This spectrum is referred to as RS6 below. The parameters were derived by comparing the available $\mathrm{H}$ Balmer lines and He lines in the spectrum with a detailed grid of LTE H/He model atmospheres and synthetic spectra. The uncertainties quoted by Saffer et al. (1994) are conservative and include the effects of external errors. His estimates are consistent with those of O'Donoghue et al. (1998) who followed a very similar approach using an independent grid of pure $\mathrm{H}$ model atmospheres in LTE. O'Donoghue et al. (1998) proposed two possible solutions from two different observations using different instrumental configurations. They found $T_{\text {eff }}=35900 \pm 250 \mathrm{~K}, \log g=5.90 \pm 0.05$ (formal errors of the fit), and $T_{\text {eff }}=34800 \pm 300 \mathrm{~K}, \log g=5.82 \pm 0.07$. Of course, they could not estimate the helium abundance with their pure $\mathrm{H}$ models. The differences here can be taken as estimates of the external errors in the O'Donoghue et al. (1998) procedure.

For their part, Telting \& Østensen (2004) found average values of $T_{\text {eff }}=34800 \mathrm{~K}, \log g=5.81$, and $\log N(\mathrm{He}) / N(\mathrm{H})=$ -1.65 , again using LTE $\mathrm{H} / \mathrm{He}$ model atmospheres. They also carried out an interesting experiment in which they fitted the instantaneous spectra of PG 1325+101 obtained during the course of time-resolved low-resolution spectroscopy, to derive apparent effective temperature and surface gravity variations of amplitude $610 \mathrm{~K}$ and 0.05 dex, respectively. No discussion of the expected uncertainties attached to the proposed average values for the atmospheric parameters was given in Telting \& Østensen (2004), but reasonable estimates of these are $\Delta T_{\mathrm{eff}} \sim 390 \mathrm{~K}$, $\Delta \log g \sim 0.05$, and $\Delta \log N(\mathrm{He}) / N(\mathrm{H}) \sim 0.02$ (external errors).

The available estimates of the atmospheric parameters (three independent sources) are all consistent with each other and point to a star with a relatively high value of the surface gravity. Despite this agreement, we felt that it would be important to obtain additional measurements because of the key role that spectroscopic constraints may play in discriminating between various asteroseismological solutions (see Charpinet et al. 2005a,b, for examples of this). To this end, we first obtained an optical spectrum of PG 1325+101 with $\sim 6 \AA$ resolution and $S / N \sim 80$ covering the range from $3060 \AA$ to $5240 \AA$ at the Steward Observatory's $2.3 \mathrm{~m}$ Telescope. This is similar to the older spectrum obtained by Saffer et al. (1994) except for the much improved $S / N$ ratio. We will refer to this newer spectrum as PB6. We also obtained at the same telescope five spectra at lower resolution $(\sim 9.4 \AA)$ covering the range from $3620 \AA$ to $6900 \AA$ which, when coadded and median-filtered, produced a spectrum of very high precision $(S / N \sim 280$ per pixel, with 3.6 pixels per resolution element). We will refer to this one as BG9. Finally, our best measurement was obtained using the Blue Spectrograph of the $6.5 \mathrm{~m}$ MMT Telescope. Four $\sim 1 \AA$ resolution spectra of PG 1325+101 were coadded to achieve a combined $S / N \sim 200$ (per pixel, with 3.0 pixels per resolution element). The latter spectrum covers the range from $\sim 4000 \AA$ to $\sim 4950 \AA$ and will be referred to as MMT1.

We analyzed these new time-averaged spectra of PG 1325+101 with the help of two detailed grids of model atmospheres, one in LTE and the other in NLTE, especially designed for subdwarf B stars. Some properties of these models are discussed in Charpinet et al. (2005a), and further details will be provided in Green et al. (in preparation). Suffice it to say here that these banks of atmosphere models and synthetic spectra were computed recently with the help of the public codes TLusty and Synspec (Hubeny \& Lanz 1995; Lanz \& Hubeny 1995). One of the conclusions of Green et al. is 

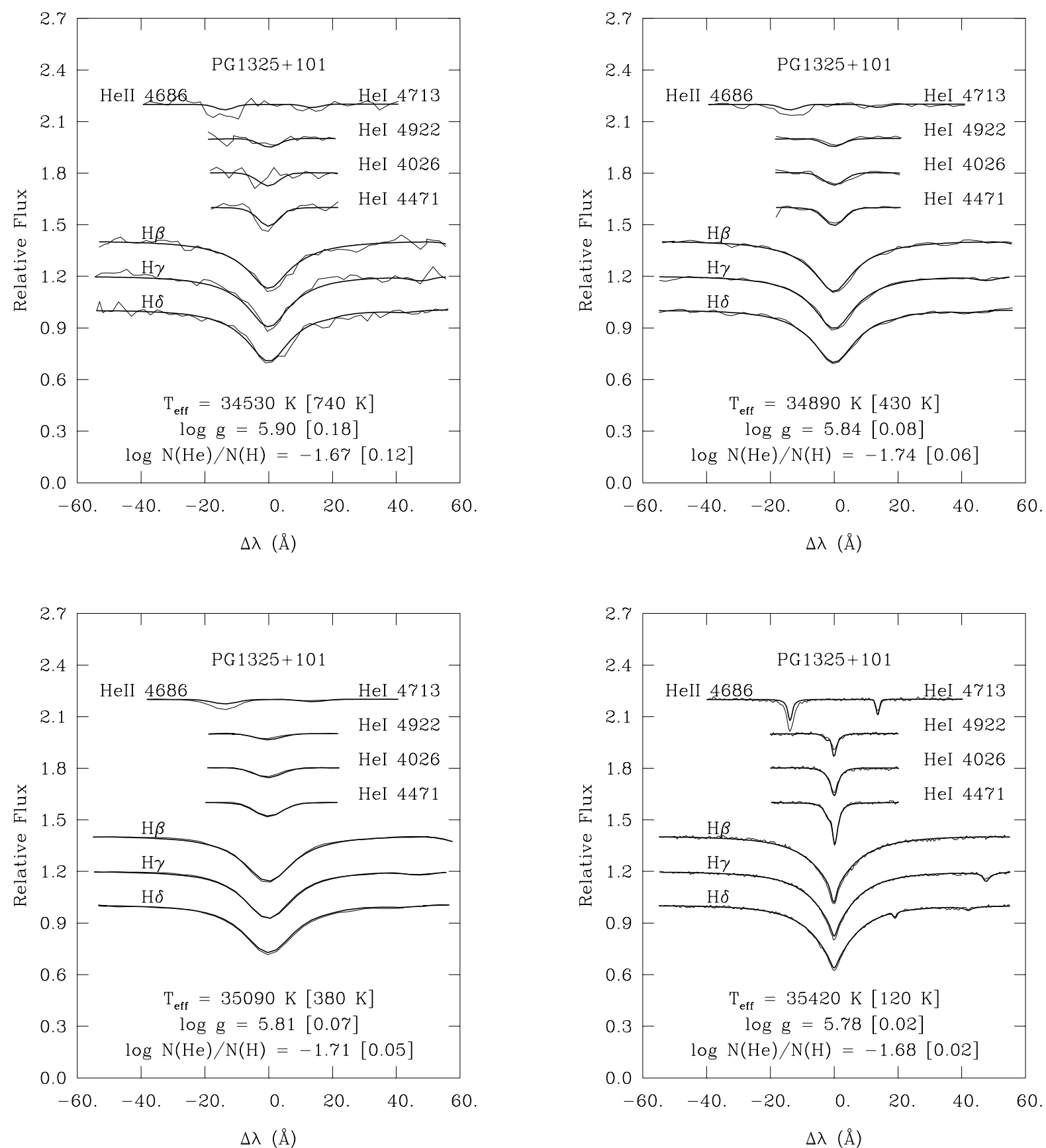

Fig. 1. Model fits (thick curves) to the hydrogen and helium lines (thin curves) available in the 4000-4950 $\AA$ spectral range in our time-averaged spectrum RS6 (upper left panel), PB6 (upper right panel), BG9 (lower left panel), and MMT1 (lower right panel).

that systematic differences due to the inclusion of higher order Balmer lines in the fits, compared to fits using only $\mathrm{H} \beta, \mathrm{H} \gamma$, and $\mathrm{H} \delta$, are quite small as long as the $S / N$ of the data is relatively high over all of the fitted lines. For this paper, we analysed all of our new spectra over the shorter spectral range defined by the MMT1 spectrum, 4000-4950 Å, for consistency.

Some of our results are shown in Fig. 1, where the quoted uncertainties are the formal errors of the fits and do not include external errors. In the upper left panel, we show our LTE solution for spectrum RS6, the original spectrum of Saffer et al. (1994), available to us since one of us (P.B.) is coauthor on that paper. While our results are consistent with the previous LTE analyses, the figure shows a rather noisy spectrum with relatively large fitting errors. The fits to our higher $S / N$ spectra are more interesting since they provide much tighter constraints on the spectroscopic solution. We illustrate the preferred NLTE fits to our new spectra in the upper right panel (PB6), in the lower left panel (BG9), and in the lower right panel (MMT1). We note that these three fits are remarkably consistent with each other and, also, are quite good. They are not perfect, however; In all three cases, the He II 4686 line is stronger than predicted. This is not new and was encountered, for example, in the pulsating EC14026 star PG 1219+534 (Charpinet et al. 2005a). This phenomenon has been referred to as the "helium problem" in hot sdB stars and the culprit is suspected to be the absence of metals in the models (see, e.g., Heber et al. 2000). Irrespective of the inherent computational problems associated with the inclusion of metals in NLTE models of sdB stars, the basic difficulty is that we do not know what metal abundances to use since their 
Table 1. Atmospheric parameters of PG 1325+101 derived from spectroscopy.

\begin{tabular}{llll}
\hline \hline Source & $T_{\text {eff }}(\mathrm{K})$ & $\log g$ & $\log N(\mathrm{He}) / N(\mathrm{H})$ \\
\hline Telting \& Østensen (2004) & $34800 \pm 390$ & $5.81 \pm 0.05$ & $-1.65 \pm 0.02$ \\
This work (PB6) & $34890 \pm 525$ & $5.84 \pm 0.09$ & $-1.74 \pm 0.07$ \\
This work (BG9) & $35090 \pm 450$ & $5.81 \pm 0.08$ & $-1.71 \pm 0.05$ \\
This work (MMT1) & $35420 \pm 390$ & $5.78 \pm 0.05$ & $-1.68 \pm 0.02$ \\
\hline Mean $^{\dagger}$ & $35050 \pm 220^{\ddagger}$ & $5.81 \pm 0.04^{\ddagger}$ & $-1.70 \pm 0.02^{\ddagger}$ \\
\hline
\end{tabular}

${ }^{\dagger}$ Reference values used for the asteroseismic analysis. ${ }^{\ddagger}$ The given error is the quadratic combination of the uncertainties of each measurement.

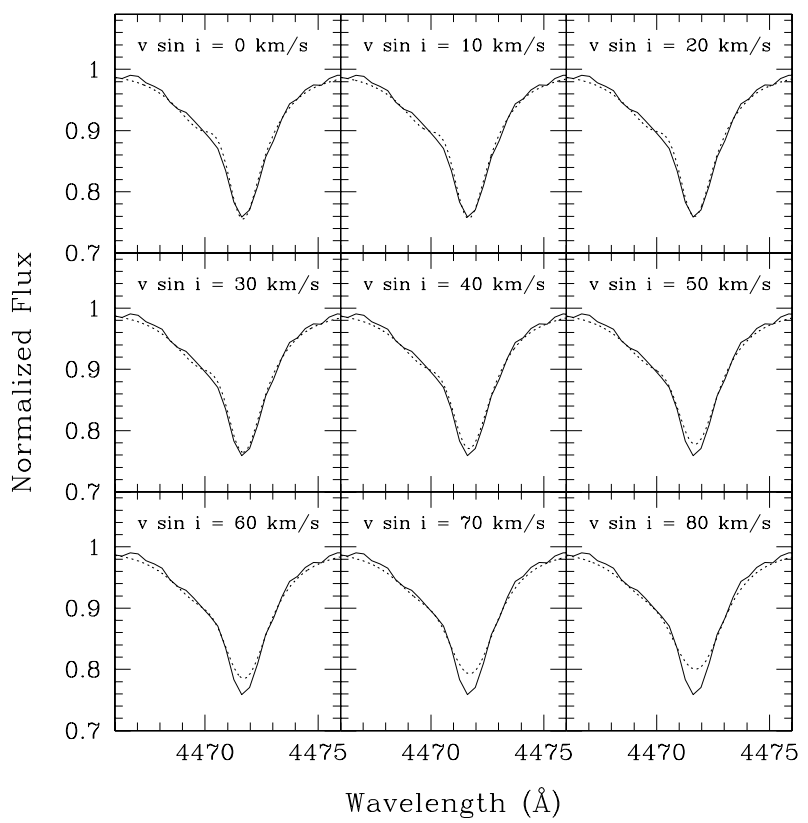

Fig. 2. Model fits (dotted curves) to the He I 4471 line (solid curves) in our MMT spectrum of PG 1325+101 assuming various values of the projected rotational velocity $V \sin i$.

atmospheric values vary from one star to another and are not in solar proportions.

Until a complete study of the spectrum of PG $1325+101$ is carried out (with feedback between derived abundances and NLTE H/He/metals models), we will have to be content with the results currently available. Taking into account external errors, which we evaluate from multiple observations with the same setup and include quadratically with the formal errors of the fits (those shown in Fig. 1), we find that our best estimates of the atmospheric parameters of PG 1325+101 are our NLTE solutions based on spectra PB6, BG9, and MMT1. The values are summarized in Table 1, and are used below, along with the estimates of Telting \& Østensen (2004), to constrain the asteroseismic solutions.

As an interesting aside, we have noticed in the past that MMT spectra similar to the one used here for PG 1325+101 are of sufficient resolution and sensitivity to reveal weak and narrow metal lines when present. This has been used quite succesfully by Chayer et al. (2003) to derive very interesting abundance patterns for several sdB stars. We therefore searched for such lines in the MMT1 spectrum in the hope of constraining $V \sin i$ from possible rotational broadening. Unfortunately, the few metal lines detectable in the MMT spectrum of PG 1325+101 are much too weak for us to use them for that purpose. No doubt such lines would be numerous in the FUV spectral range but, unfortunately, PG $1325+101$ has not been observed by FUSE, nor will it ever be. However, we noticed that the He I 4471 line is particularly well reproduced in our fit (see lower right panel of Fig. 1) and that it is relatively narrow, so that it might perhaps serve as a surrogate for metal lines.

In Fig. 2, we show the results of modeling efforts that assume a range of possible values for the projected rotational velocity. There is no sign of rotational broadening in the observed profile of the He I 4471 line, and the only useful result that we can gather from this experiment here is a limit of perhaps $20-30 \mathrm{~km} \mathrm{~s}^{-1}$ on $V \sin i$. While this is interesting in itself and suggests a relatively low rotation rate, this limit is not stringent enough to be combined with asteroseismic results, as will be discussed below. Moreover, Telting \& Østensen (2004) found, from their time-resolved spectroscopic analysis of PG $1325+101$, a radial velocity modulation of half-amplitude $\sim 18 \mathrm{~km} \mathrm{~s}^{-1}$ due to the dominant pulsation mode. In a time averaged spectrum, this would result in a pulsational broadening that would be undistinguishable from rotational broadening, unless the latter is significantly larger than the former. Hence, low values of $V \sin i$ may not be measurable at all based on time-averaged spectra.

\section{Nonadiabatic asteroseismic analysis}

\subsection{Search for the optimal model}

The moderately rich pulsational spectrum resulting from the multisite PG 1325+101 campaign reported in Paper I ranks this subdwarf B star among those particularly well suited for a detailed asteroseismic study. To achieve this objective, we followed the same procedure applied to the fast sdB pulsator PG 1219+534, which is described at length in Charpinet et al. (2005b). This procedure is a "forward modeling" method which consists of a systematic search of the best matching models within the vast ensemble of model solutions that can possibly reproduce the observed period spectrum of the star under consideration. For PG 1325+101, we based our analysis on 12 wellestablished periods out of the 15 given in the upper part of the Table 2 provided in Paper I (the $F_{n}$ frequencies). Additional suspected - but somewhat uncertain - frequencies dubbed $f_{1}-f_{15}$ and given in the lower part of this table were not used in the asteroseismic analysis that follows. This conservative approach strongly limits the risk of overly (and perhaps incorrectly) constraining the models based on signal that may turn out to be spurious. Nonetheless, it will be interesting to discuss a posteriori how these additional periodicities - some of them being possibly real oscillations of the star - may be interpreted and compared to the theoretical periods of the optimal model solution uncovered (see Sect. 3.2). Among the well-secured frequencies, three $\left(F_{6}\right.$, $F_{7}$, and $\left.F_{8}\right)$ are suspected components of a well-formed triplet, and thus must be considered as a single independent mode for the asteroseismology exercise (see, e.g., Charpinet et al. 2005a). The central frequency (period) of the triplet $\left(F_{7}\right)$ was kept as the $m=0$ component that needs to be compared to periods computed from purely spherical pulsation models (see below). 
We note that considering these three frequencies as independent modes (i.e., with different values of the indices $k$ and/or $\ell$ ) significantly degrades the period fit of the best model solution because it requires the presence of too many modes in a very narrow frequency range, which is inconsistent with the structure of the theoretical pulsation spectrum with no rotation. Finally, the period $F_{15}$ was not considered, as it clearly corresponds to the first harmonic of the dominant mode, and thus is not an eigenfrequency of the oscillating star.

Theoretical spectra to which the observed periods can be compared were computed using the so-called "second generation" models suitable for pulsating subdwarf B stars (Charpinet et al. 1997, 2001). These models are static structures extending as deep as $\log q \equiv \log \left(1-M(r) / M_{*}\right) \simeq-0.05$ that incorporate the nonuniform abundance profiles of iron predicted by the theory of microscopic diffusion assuming an equilibrium between gravitational settling and radiative levitation. Such models with diffusion are currently the most accurate structures available to derive the pulsation properties of pulsating $B$ subdwarfs, both in terms of predicting which modes are driven and of providing precise period evaluations for asteroseismology (Charpinet et al. 2005b; Fontaine et al. 2006). Four fundamental parameters are needed to fully specify the internal structure of a hot subdwarf B star with the second generation models: the effective temperature $T_{\text {eff }}$, the surface gravity $\log g$, the total mass of the star $M_{*}$, and the logarithmic fractional mass of the hydrogenrich envelope $\log q(H) \equiv \log \left[M(H) / M_{*}\right]$. The latter parameter is intimately related to the more familiar parameter $M_{\mathrm{env}}$, the mass of the H-rich envelope. These are the natural quantities that define the parameter space which needs to be explored in order to localize the optimal model(s). Pulsation properties of the models are evaluated through the combined application of adiabatic and nonadiabatic nonradial pulsation codes described in Brassard et al. (1992) and Fontaine \& Brassard (1994), respectively. These provide the necessary quantities to compare with the seismic observations which, for asteroseismology purposes, are mainly the periods and the stability coefficients. The pulsation calculations were performed assuming perfect spherical symmetry, as is standard and fully justified for slowly rotating stars. The comparison is achieved using a period matching code that derives the best possible match for that particular model. This match is evaluated quantitatively in a minimum dispersion sense, through the computation of a merit function

$S^{2}=\sum_{i=1}^{n}\left(P_{\mathrm{obs}}^{i}-P_{\mathrm{th}}^{i}\right)^{2}$,

where $P_{\mathrm{obs}}^{i}$ is one of the $n$ periodicities observed (in the present case, $n=12$ ) and $P_{\text {th }}^{i}$ is the theoretical period that matches it best (note that we used the weights $\sigma_{i}=\sigma=1$ in the evaluation of $S^{2}$; see Eq. (1) of Charpinet et al. 2005b). Since no a priori mode identification is available for the observed periods (as is generally the case for pulsating sdB stars), this last step is a global procedure that seeks the best possible simultaneous fit of the observed periods. The mode identification then emerges as a result of this procedure.

We carried out a search for optimal model solution(s) by first applying a dedicated optimization code based on a Genetic Algorithm (GA), designed to efficiently explore the vast model parameter space. This code is aimed at finding minima for the quantity $S^{2}\left(T_{\text {eff }}, \log g, \log q(H), M_{*}\right)$ expressed as a function of the model parameters. The value of $S^{2}$ for a given set of parameters is derived from the period matching procedure referred to above. However, as will be illustrated and justified below, due to the nature of some degeneracies that affect potential solutions, we initially imposed the additional constraint of keeping the parameter $T_{\text {eff }}$ constant, set to the value dictated by spectroscopy. To this end, we adopted the mean value of $T_{\text {eff }}=35050 \mathrm{~K}$ $( \pm 220 \mathrm{~K})$ as a reasonable estimate of the effective temperature of PG 1325+101 according to the four independent measurements summarized in Table 1 (excluding here the cruder estimate obtained from the low- $S / N$ spectrum RS6). Hence, the search was initially limited to the three-dimensional space defined by the remaining free fundamental parameters.

Initial boundaries considered for the search were defined as follows: $5.65 \leq \log g \leq 6.05-$ i.e., loosely set according to current spectroscopic estimates of $\log g$ for PG $1325+101-$, $-5.0 \leq \log q(H) \leq-2.0$, and $0.30 \leq M_{*} / M_{\odot} \leq 0.70$, based on current constraints provided by Extreme Horizontal Branch stellar evolution and various formation scenarios (see Charpinet et al. 2005b, for details). For the pulsation calculation step, we considered modes of degree $\ell=0$ up to $\ell=4$ with periods in the range 60-500 s, i.e., covering amply the range of periods observed in PG 1325+101. The inclusion of $\ell>2$ oscillations was motivated by the density of modes in the observed period range that simply cannot be explained in terms of low degree $(\ell \leq 2)$ modes only, unless one invokes an improbable large frequency splitting caused by a hypothetical rapid differential rotation of the star, as proposed by Kawaler \& Hostler (2005), which is tuned to mimic the typical p-mode spectrum of a spherical star with higher degree modes involved. In the current analysis, we favor the simpler explanation that modes of degree higher than $\ell=2$ are effectively seen in this star. Contrary to previously published asteroseismic studies which were limited to $\ell=3$ modes, $\ell=4$ pulsations are also included here. While not required by the mode density argument, according to recent calculations (e.g., Randall et al. 2005; Jeffery et al. 2005), their visibility is comparable, or even superior, to the $\ell=3$ modes in the optical wavelength domain. Hence, considering pulsations with $\ell=3$ while rejecting $\ell=4$ would overly restrict the parameter space for the search of a best-fit solution. In addition, evidence for the presence of $\ell=4$ modes in two EC14026 pulsators has been suggested by Jeffery et al. (2004, 2005).

Within the search domain specified, the GA identified one family of solutions that turns out to be highly consistent with the independent spectroscopic estimates of the atmospheric parameters of PG $1325+101$. At fixed $T_{\text {eff }}=35050 \mathrm{~K}$, this bestfit model solution has parameters $\log g=5.8105$ (while estimations from spectroscopy lead to a mean value of $\log g=$ $5.81 \pm 0.04), \log q(H)=-4.2747$, and $M_{*}=0.4988 M_{\odot}$. With a $S^{2}$ value of 4.89 (or $\log S^{2}=0.69$ ), this model provides an excellent simultaneous match to the 12 periods observed in this star (see below). We next examined the shape of the $S^{2}$ hypersurface in the vicinity of this best-fit solution, exploring, this time, the full $4 \mathrm{~d}$-space. This includes the effects of varying the effective temperature in the broad interval $30000 \mathrm{~K} \leq T_{\text {eff }} \leq 38000 \mathrm{~K}$. Maps displayed in Fig. 3 summarize the gist of our results. They show the complex shape of the $S^{2}$-function (shown as isocontours of constant value of $\log S^{2}$ ) in the vicinity of the potential solution, whose exact location according to the GA-code is indicated by a red-cross. These maps respectively show slices of this function along the $\log g-T_{\text {eff }}$ plane (at fixed parameters $\log q(H)$ and $M_{*}$, which are set to their optimal values) and along the $M_{*}-\log q(H)$ plane (at fixed parameters $T_{\text {eff }}$ and $\log g$, set to their optimal values). Best fitting models corresponding to low values of $S^{2}$ appear as dark blue regions, while red areas indicate regions of the model parameter space where theoretical periods computed from these models do not fit the observed 

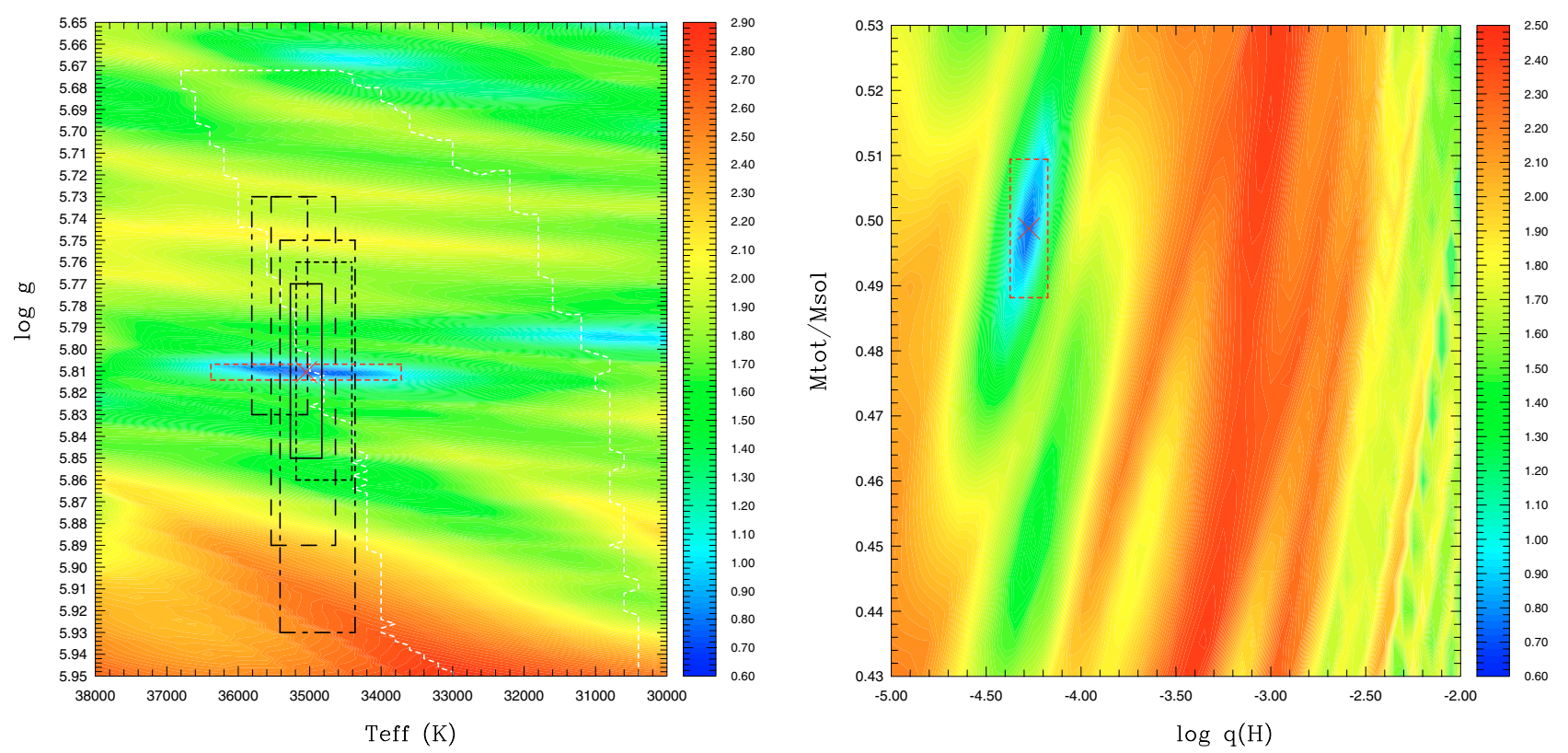

Fig. 3. Left panel: slice of the $S^{2}$-function (in logarithmic units) along the $\log g-T_{\text {eff }}$ plane at fixed parameters $M_{*}$ and $\log q(H)$, set to their optimal values found from the best-fit model solution $\left(M_{*}=0.4988 M_{\odot}\right.$ and $\left.\log q(H)=-4.2747\right)$. The black dot-dot-dashed-line, dashed-line, dot-dashed-line, and dotted-line rectangles represent the various spectroscopic estimates and their uncertainties for the atmospheric parameters of PG 1325+101. They correspond to the MMT1, BG9, PB6, and Telting \& Østensen (2004) measurements, respectively. The mean values derived from these measurements appear as the black solid-line rectangle. The red dotted-line rectangle shows the estimated error box associated with our asteroseismic determination of the parameters $\log g$ and $T_{\text {eff }}$. The red cross indicates the position of the best-fit solution. The white dottedline delimits the region in the $\log g-T_{\text {eff }}$ plane where all the observed periods are identified with modes predicted to be unstable according to nonadiabatic theory. Right panel: slice of the $S^{2}$-function (in logarithmic units) along the $M_{*}-\log q(H)$ plane at fixed parameters $T_{\text {eff }}$ and $\log g$, set to their optimal values found from the best-fit model solution $\left(T_{\text {eff }}=35,050 \mathrm{~K}\right.$ and $\left.\log g=5.8105\right)$. The red dotted-line rectangle shows the estimated error box associated with our asteroseismic determination of the parameters $\log g$ and $T_{\text {eff. }}$ The red cross indicates the position of the best-fit solution.

periods well (high values of $S^{2}$ ). Considering the logarithmic scale used to represent the merit function on these plots, we stress that the blue regions correspond to well-defined minima.

Thorough exploration of the model parameter space with the GA code revealed the presence of other potential families of solutions having comparable quality of the period fit. These, however, are found at much lower surface gravities $(\log g \simeq 5.70$ and below), i.e., in obvious conflict with all present measurements of $\log g$ provided by spectroscopy (see Fig. 3), and are clearly rejected on this basis. Similar degeneracies have been encountered in all EC14026 stars analysed so far, and hence accurate spectroscopic measurements of the atmospheric parameters have proven essential to limit duplicities in the asteroseismic solutions. However, another kind of degeneracy appears when a change in one of the model parameters can be almost exactly compensated by a change in another model parameter, such that the computed periods remain unchanged. Such a phenomenon was encountered in the study of PG 1219+534 (Charpinet et al. $2005 \mathrm{~b}$ ), and indeed occurs in a similar way in the present analysis of PG $1325+101$.

First, there is a weak correlation between the $\log q(H)$ and $T_{\text {eff }}$ parameters. A change of $\log q(H)$ relative to its optimal value while keeping the parameter $M_{*}$ constant (set to its optimal value) generates a shift of the position of the local minimum, mostly along the $T_{\text {eff }}$ axis. This trend is illustrated in the map shown in Fig. 4 which represents the "projection" of the $\log q(H)$ axis onto the $\log g-T_{\text {eff }}$ plane. More precisely, the logarithm of the $S^{2}$ value associated with each grid point shown on this map is the minimum value found among all the values of the $S^{2}$-function obtained at fixed $T_{\text {eff }}, \log g$ (with values corresponding to the specific grid point), and $M_{*}$ (set to its optimal value), but with the parameter $\log q(H)$ varying within the limits of the specified search domain, i.e., between $\log q(H)=-5.0$ and -2.0 . The labelled axis positioned along the valley associated with the preferred solution indicates the position of the local minimum of $S^{2}$ as a function of $\log q(H)$ near the optimal solution. There is a clear monotonic trend showing that this minimum shifts from higher to lower $T_{\text {eff }}$ as the envelope mass of the star increases (i.e., the value of $\log q(H)$ increases). However, this shift is also associated with a degradation of the overall quality of the period fit as the $S^{2}$-value of the minimum increases as well. Consequently, the optimal solution still occupies the center of a well defined region of the parameter space.

This is no longer the case when one explores changes applied to the total mass of the star. A similar map was constructed to visualize the "projection" of the $M_{*}$-axis onto the $\log g-T_{\text {eff }}$ plane (Fig. 5). The parameter $\log q(H)$ was kept constant, set to its optimal value, and the total mass was varied between 0.40 and $0.60 M_{\odot}$ (i.e., a subset of the search domain relevant for the present illustration). The map clearly shows that a correlation exists between the parameters $M_{*}, T_{\text {eff }}$, and to a much lesser extent, $\log g$. A change in $M_{*}$ generates a shift in both $T_{\text {eff }}$ and $\log g$ of the position of the $S^{2}$ minimum (as indicated by the labelled axis along the region of best-fit models). However, contrary to the case of the $\log q(H)$ parameter previously discussed, there is no degradation of the quality of the period fit over the range considered for the $M_{*}$ parameter. This leads to a linedegeneracy, clearly apparent in Fig. 5 as a long and flat valley of minimum $S^{2}$, along which models reproduce similarly well the observed periods. As already observed for PG $1219+534$, the trend is monotonic and associates cooler $T_{\text {eff }}$ (and slightly lower $\log g$ ) to lower values of $M_{*}$. The correlation with the effective 


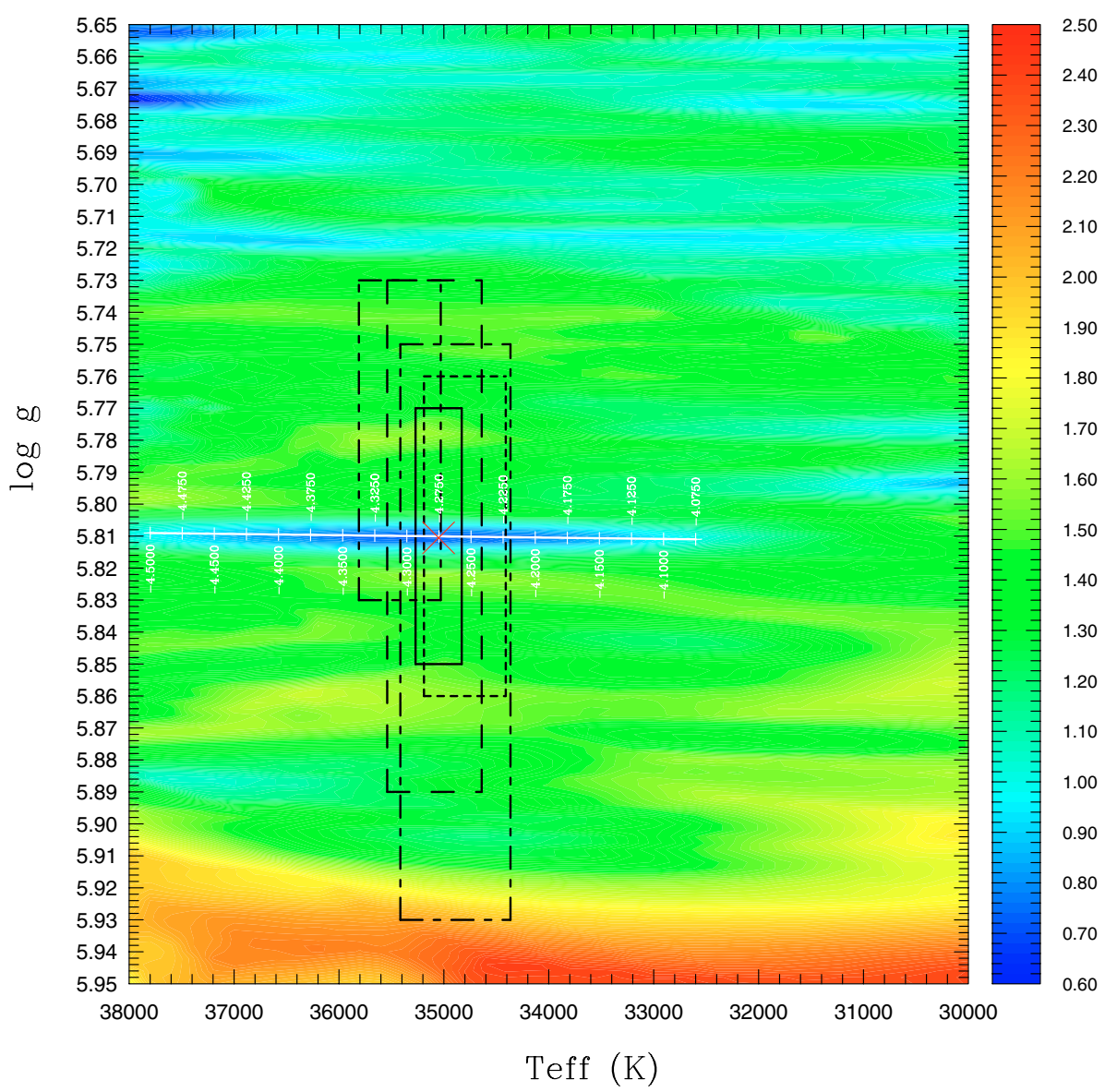

Fig. 4. Slice of the "projected" $S^{2}$-function (in logarithmic units) along the $\log g-T_{\text {eff }}$ plane at fixed parameter $M_{*}$, set to its optimal value from the best-fit model solution $\left(M_{*}=0.4988 M_{\odot}\right)$. The projected $\log q(H)$ parameter was varied between -2.0 and -5.0 , in steps of 0.025 . The labelled axis positioned along the valley of minimum $S^{2}$ indicates the location of the local minimum of $S^{2}$ for the given values of $\log q(H)$. The dot-dotdashed-line, dashed-line, dot-dashed-line, and dotted-line rectangles represent the various spectroscopic estimates with their uncertainties for the atmospheric parameters of PG 1325+101. They correspond to the MMT1, BG9, PB6, and Telting \& Østensen (2004) measurements, respectively. The mean values derived from these measurements appear as the solid-line rectangle.

temperature is however much stronger than the (weak) correlation with the surface gravity parameter. Again, the additional constraints brought by spectroscopy are essential to lift this degeneracy. The measurements of $T_{\text {eff }}$, with their associated errors, permits us to select the appropriate section along the line of degeneracy which correspond to the "correct" solution. The fact that one has to rely on the spectroscopic value of the effective temperature to uniquely derive the total mass of the star from asteroseismology indicates, however, that $M_{*}$ cannot be measured independently of $T_{\text {eff }}$ for PG $1325+101$. Finally, we stress that the existence of this line-degeneracy justifies a posteriori the strategy of setting the value of $T_{\text {eff }}$ according to spectroscopic estimates during the initial search with the GA-code. Not providing this additional constraint would have potentially led the code to converge anywhere along this extended and unbounded region of minimum $S^{2}$.

\subsection{Period fit and mode identification}

The optimal model isolated for PG $1325+101$ provides an excellent match to the 12 independent periods clearly identified in this star. The resulting identification of the modes involved in the luminosity variations is given in Table 2 (see also Fig. 6 for a graphical representation). Besides the quantities that reflect the properties of the nonradial modes computed for the best-fit model (we again refer the reader to Charpinet et al. 2005b for a complete description), Table 2 provides the derived distribution of the observed periods $\left(P_{\mathrm{obs}}\right)$ as they were matched to the theoretical modes with periods $P_{\text {th }}$, degree $\ell$ and radial order $k$. The relative and absolute differences in period, $\Delta P / P$ (in \%) and $\Delta P$ (in seconds), for each pair $\left(P_{\mathrm{obs}}, P_{\mathrm{th}}\right)$ is also given in this table.

The average relative dispersion between the fitted periods is $\overline{\Delta P / P} \simeq 0.46 \%$. On an absolute scale, this represents an average dispersion of $\overline{\Delta P} \simeq 0.54 \mathrm{~s}$ ( or $\overline{\Delta v} \simeq 40.46 \mu \mathrm{Hz}$ ), with, for the worst case, a difference of only $\Delta P \simeq 1.19 \mathrm{~s}$. The quality of this simultaneous fit of all the periods is similar to - even slightly better than - those obtained for other EC14026 pulsators analysed under comparable conditions. Yet, the accuracy at which the periods are measured is still approximately one order of magnitude better than the mean period dispersion achieved for the fit. This difference was already noted in previous studies and it points to the fact that current equilibrium models describing the structure of sdB stars still suffer from imperfections that leave significant room for improvements. Such improvements are, of course, one of the goals to be sought in future asteroseismic studies of $\mathrm{sdB}$ pulsators.

The observed periods are identified with radial $(\ell=0)$ and nonradial $(\ell=1-4) p$-modes having low radial orders $(k=1-5)$. All but one mode are indeed predicted to be excited by the "Fe" $\kappa$-mechanism according to nonadiabatic calculations 


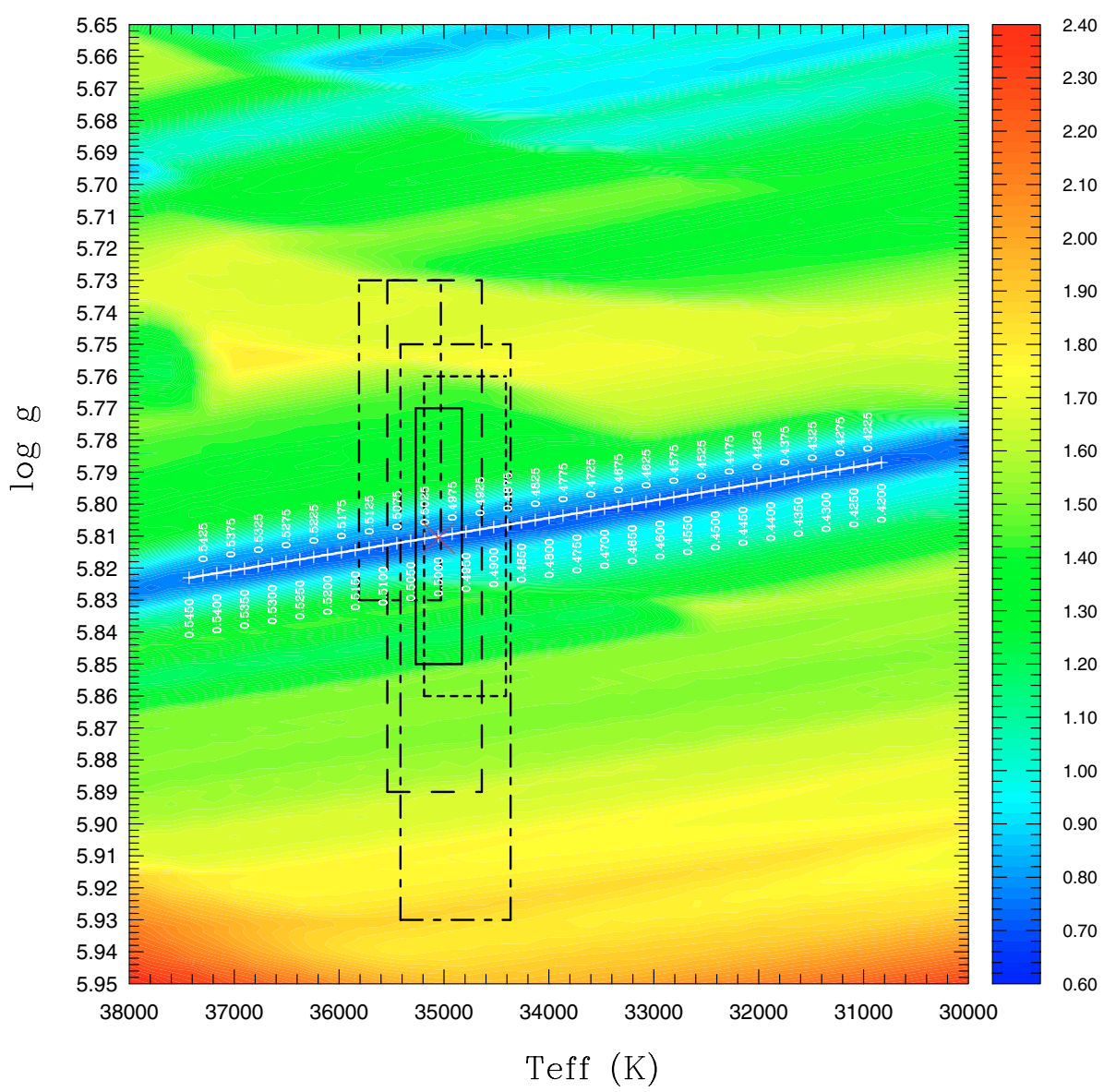

Fig. 5. Slice of the "projected" $S^{2}$-function (in logarithmic units) along the $\log g-T_{\text {eff }}$ plane at fixed parameter $\log q(H)$ set to its optimal value found for the best-fit model solution $(\log q(H)=-4.2747)$. The projected, $M_{*}$ parameter was varied between $0.40 M_{\odot}$ and $0.60 M_{\odot}$ (by steps of $0.0025 M_{\odot}$ ). The labelled axis positioned along the valley of minimum $S^{2}$ indicates the location of the local minimum of $S^{2}$ for the given values of $M_{*}$. The solid-line, dashed-line, dot-dashed-line, and dotted-line rectangles represent the various spectroscopic estimates and their uncertainties for the atmospheric parameters of PG 1325+101. They correspond to the MMT1, BG9, PB6, and Telting \& Østensen (2004) measurements, respectively.

(i.e., negative values of $\sigma_{\mathrm{I}}$ in Table 2). The exception is the period $F_{1}(167.778 \mathrm{~s})$ associated to the stable $\ell=1, k=1$ mode. We find, however, that this mode is only marginally stable and a slight decrease of only $\sim 40 \mathrm{~K}$ of the effective temperature of the model, which is well within the uncertainties for the determination of this parameter, is sufficient for the mode to become unstable. Indeed, the region of the $\log g-T_{\text {eff }}$ plane where all the observed periods can be associated to driven modes is shown in Fig. 3 (delimited by the white dotted-line). Clearly, the relatively high $T_{\text {eff }}$ estimated for PG $1325+101$ places this star close to the blue edge of this region. But it remains entirely compatible, within the uncertainties, with the nonadiabatic stability calculations. Hence, PG $1325+101$ is yet another example for which strong consistency can be achieved between three independent aspects of the modeling of these stars: 1) the reproduction of the observed period distribution; 2) the nonadiabatic properties of the pulsations; and 3) the determination of the atmospheric parameters through spectroscopy. However, at this point we stress that several modes predicted to be excited in the model are in fact not detected in the present data. Such "holes" (or "missing modes") in pulsation period spectra of rapidly pulsating B subdwarfs are common, and remain largely unexplained so far. Nonetheless, since our past experience indicates that high $S / N$ ratio photometric observations of EC14026 stars usually lead to the detection of more pulsation modes, it is likely that not all modes in the excited frequency bandpass are driven up to sufficient amplitudes such that they can easily be seen. Large intrinsic, and possibly time-variable, amplitude differences may also exist between excited modes. Since the standard, linear oscillation theory is incapable of predicting mode amplitudes, a nonlinear approach to the nonadiabatic pulsation problem will be essential to fully understand such behavior. Unfortunately, such a nonlinear nonradial pulsation theory does not yet exist.

We note also that the period of $129.893 \mathrm{~s}\left(F_{7}\right)$, possibly a triplet according to the data, is indeed identified as a $\ell=1$ mode in the fit. The dominant mode, $F_{4}$, corresponding to a period of $137.826 \mathrm{~s}$, is associated with a $\ell=2$ mode in the present fit, contrary to the suggestion of Telting \& Østensen (2004) that it could be a radial pulsation (although they admit that other identifications might be possible). However, considering the global mode distribution observed in PG 1325+101, as was done in this analysis, it appears difficult to assign this period to a radial mode. In this context, the presence of residual power in the Fourier spectrum near $F_{4}$ (see Paper I) may suggest the existence of fine structure associated with this frequency, which could also indicate a nonradial mode (see below).

At this stage, it is interesting to look back at Table 2 of Paper I. The periodicities $f_{1}-f_{15}$ given in the lower part of this table are all considered questionable and, as such, were ignored in the preceding asteroseismic analysis. Some of these 
Table 2. Pulsation properties of the best-fit model solution and mode identification.

\begin{tabular}{|c|c|c|c|c|c|c|c|c|c|}
\hline$l$ & $k$ & $\begin{array}{l}P_{\text {obs }} \\
(\mathrm{s})\end{array}$ & $\begin{array}{l}P_{\text {th }} \\
(\mathrm{s})\end{array}$ & $\begin{array}{c}\sigma_{\text {I }}(\text { stability }) \\
(\mathrm{rad} / \mathrm{s})\end{array}$ & $\begin{array}{l}\log E \\
\text { (erg) }\end{array}$ & $C_{k l}$ & $\begin{array}{c}\Delta P / P \\
(\%)\end{array}$ & $\begin{array}{l}\Delta P \\
(\mathrm{~s})\end{array}$ & Comments \\
\hline 0 & 7 & $\ldots$ & 70.810 & $+3.754 \times 10^{-5}(\mathrm{~S})$ & 39.664 & $\ldots$ & $\ldots$ & $\ldots$ & \\
\hline 0 & 6 & $\ldots$ & 77.096 & $-3.398 \times 10^{-5}(\mathrm{U})$ & 40.024 & $\ldots$ & $\ldots$ & $\ldots$ & \\
\hline 0 & 5 & $\ldots$ & 86.656 & $-8.407 \times 10^{-5}(\mathrm{U})$ & 40.087 & $\cdots$ & $\cdots$ & $\cdots$ & \\
\hline 0 & 4 & 99.808 & 99.841 & $-4.361 \times 10^{-5}(\mathrm{U})$ & 40.474 & $\ldots$ & -0.03 & -0.032 & $F_{12} ;\left[f_{15}\right.$ closeby (see text) $]$ \\
\hline 0 & 3 & $\ldots$ & 109.044 & $-2.109 \times 10^{-5}(\mathrm{U})$ & 40.758 & $\ldots$ & $\ldots$ & $\ldots$ & \\
\hline 0 & 2 & $\ldots$ & 131.753 & $-5.736 \times 10^{-6}(\mathrm{U})$ & 41.080 & $\ldots$ & $\ldots$ & $\ldots$ & \\
\hline 0 & 1 & {$[150.615]$} & 150.218 & $-1.865 \times 10^{-7}(\mathrm{U})$ & 42.142 & $\cdots$ & {$[+0.26]$} & {$[+0.397]$} & {$\left[f_{1}\right]$} \\
\hline 0 & 0 & $\ldots$ & 168.298 & $+1.372 \times 10^{-8}(\mathrm{~S})$ & 42.190 & $\ldots$ & $\ldots$ & $\ldots$ & \\
\hline 1 & 8 & $\ldots$ & 70.047 & $+4.800 \times 10^{-5}(\mathrm{~S})$ & 39.680 & 0.0053 & $\ldots$ & $\ldots$ & \\
\hline 1 & 7 & $\ldots$ & 75.884 & $-2.992 \times 10^{-5}(\mathrm{U})$ & 39.932 & 0.0064 & $\ldots$ & $\ldots$ & \\
\hline 1 & 6 & $\begin{array}{l}\cdots \\
\cdots\end{array}$ & 85.932 & $-8.431 \times 10^{-5}(\mathrm{U})$ & 40.068 & 0.0065 & $\cdots$ & $\cdots$ & \\
\hline 1 & 5 & 98.379 & 97.755 & $-3.903 \times 10^{-5}(\mathrm{U})$ & 40.512 & 0.0121 & +0.64 & +0.625 & $F_{13}$ \\
\hline 1 & 4 & 106.608 & 107.214 & $-3.035 \times 10^{-5}(\mathrm{U})$ & 40.612 & 0.0110 & -0.57 & -0.606 & $F_{10}$ \\
\hline 1 & 3 & 129.893 & 130.506 & $-5.932 \times 10^{-6}(\mathrm{U})$ & 41.080 & 0.0140 & -0.47 & -0.614 & $F_{7} ; F_{6}, F_{8}$ closeby $(\sim 7 \mu \mathrm{Hz})$ \\
\hline 1 & 2 & 144.983 & 145.108 & $-4.356 \times 10^{-7}(\mathrm{U})$ & 41.909 & 0.0261 & -0.09 & -0.125 & $F_{3} ;\left[f_{2}\right.$ closeby $\left.(\sim 6 \mu \mathrm{Hz})\right]$ \\
\hline 1 & 1 & 167.778 & 167.702 & $+1.033 \times 10^{-8}(\mathrm{~S})$ & 42.150 & 0.0175 & +0.04 & +0.076 & $F_{1}$; only marginally stable \\
\hline 2 & 7 & $\ldots$ & 74.423 & $-2.051 \times 10^{-5}(\mathrm{U})$ & 39.804 & 0.0068 & $\ldots$ & $\ldots$ & \\
\hline 2 & 6 & $\ldots$ & 84.695 & $-7.876 \times 10^{-5}(\mathrm{U})$ & 40.062 & 0.0088 & $\ldots$ & $\ldots$ & \\
\hline 2 & 5 & 94.830 & 94.073 & $-3.866 \times 10^{-5}(\mathrm{U})$ & 40.493 & 0.0189 & +0.80 & +0.757 & $F_{14}$ \\
\hline 2 & 4 & $\ldots$ & 105.229 & $-4.172 \times 10^{-5}(\mathrm{U})$ & 40.484 & 0.0129 & $\ldots$ & $\ldots$ & \\
\hline 2 & 3 & $\cdots$ & 126.399 & $-5.323 \times 10^{-6}(\mathrm{U})$ & 41.168 & 0.0376 & $\cdots$ & $\cdots$ & \\
\hline 2 & 2 & 137.826 & 137.597 & $-2.066 \times 10^{-6}(\mathrm{U})$ & 41.398 & 0.0400 & +0.17 & +0.229 & $F_{4} ;\left[f_{5}-f_{10}\right.$ closeby $($ see text $\left.)\right]$ \\
\hline 2 & 1 & $\ldots$ & 166.486 & $+6.768 \times 10^{-10}(\mathrm{~S})$ & 42.090 & 0.0224 & $\ldots$ & $\ldots$ & \\
\hline 2 & 0 & $\ldots$ & 204.017 & $+2.018 \times 10^{-10}(\mathrm{~S})$ & 45.108 & 0.4340 & $\ldots$ & $\ldots$ & \\
\hline 3 & 7 & $\ldots$ & 72.984 & $-1.561 \times 10^{-6}(\mathrm{U})$ & 39.698 & 0.0094 & $\ldots$ & $\ldots$ & \\
\hline 3 & 6 & $\ldots$ & 82.331 & $-5.810 \times 10^{-5}(\mathrm{U})$ & 40.108 & 0.0211 & $\ldots$ & $\ldots$ & \\
\hline 3 & 5 & $\ldots$ & 89.796 & $-5.629 \times 10^{-5}(\mathrm{U})$ & 40.297 & 0.0268 & $\ldots$ & $\ldots$ & \\
\hline 3 & 4 & $\ldots$ & 103.171 & $-4.911 \times 10^{-5}(\mathrm{U})$ & 40.419 & 0.0203 & $\ldots$ & $\ldots$ & \\
\hline 3 & 3 & 117.859 & 117.427 & $-5.947 \times 10^{-6}(\mathrm{U})$ & 41.218 & 0.0794 & +0.37 & +0.432 & $F_{9}$ \\
\hline 3 & 2 & 134.570 & 133.376 & $-4.617 \times 10^{-6}(\mathrm{U})$ & 41.134 & 0.0299 & +0.89 & +1.194 & $F_{5} ;\left[f_{11}, f_{12}\right.$ closeby (see text) $]$ \\
\hline 3 & 1 & $\ldots$ & 163.198 & $-3.627 \times 10^{-8}(\mathrm{U})$ & 42.041 & 0.0701 & $\ldots$ & $\ldots$ & \\
\hline 3 & 0 & $\ldots$ & 175.018 & $+9.313 \times 10^{-9}(\mathrm{~S})$ & 43.021 & 0.1849 & $\ldots$ & $\ldots$ & \\
\hline 4 & 7 & $\ldots$ & 71.627 & $+2.344 \times 10^{-5}(\mathrm{~S})$ & 39.652 & 0.0136 & $\ldots$ & $\ldots$ & \\
\hline 4 & 6 & $\ldots$ & 78.926 & $-3.765 \times 10^{-5}(\mathrm{U})$ & 40.121 & 0.0332 & $\ldots$ & $\ldots$ & \\
\hline 4 & 5 & $\cdots$ & 87.125 & $-7.648 \times 10^{-5}(\mathrm{U})$ & 40.130 & 0.0214 & $\cdots$ & $\cdots$ & \\
\hline 4 & 4 & 99.823 & 100.752 & $-4.850 \times 10^{-5}(\mathrm{U})$ & 40.423 & 0.0323 & -0.93 & -0.929 & $F_{11} ;\left[f_{15}\right.$ closeby (see text) $]$ \\
\hline 4 & 3 & $\ldots$ & 110.825 & $-1.375 \times 10^{-5}(\mathrm{U})$ & 40.919 & 0.0618 & $\ldots$ & $\ldots$ & \\
\hline 4 & 2 & $\cdots$ & 131.468 & $-5.626 \times 10^{-6}(\mathrm{U})$ & 41.074 & 0.0254 & $\begin{array}{l}\cdots \\
\cdots\end{array}$ & $\begin{array}{l}\cdots \\
\cdots\end{array}$ & \\
\hline 4 & 1 & 156.518 & 157.317 & $-1.004 \times 10^{-7}(\mathrm{U})$ & 42.073 & 0.1159 & -0.51 & -0.799 & $F_{2}$ \\
\hline 4 & 0 & $\ldots$ & 169.420 & $+1.786 \times 10^{-8}(\mathrm{~S})$ & 42.391 & 0.0703 & $\ldots$ & $\ldots$ & \\
\hline
\end{tabular}

periodicities may however correspond to real pulsations of the star and a comparison a posteriori with the theoretical periods and mode identification associated with our optimal model solution could provide further insight. All values and comments given within brackets in Table 2 are related to these uncertain frequencies.

An obvious correspondence exists between the $f_{1}$ periodicity $(P=150.615 \mathrm{~s})$ and the unassigned $\ell=0, k=1$ theoretical mode $(P=150.218 \mathrm{~s})$. This match, with a relative dispersion of $\Delta P / P=+0.26 \%(\Delta P=+0.397 \mathrm{~s}$ on an absolute scale $)$, does not degrade the overall period fit and suggests, at the outset, that $f_{1}$ may be a real oscillation mode of PG $1325+101$. On the other hand, we find no equivalent in the optimal seismic model for the two close and apparently isolated frequencies $f_{3}$ and $f_{4}$. Test calculations indicate that modes of degree $\ell=6$ or more would have to be invoked to achieve a satisfactory match with one of these frequencies, which sounds somewhat improbable. It is however difficult to assess whether these relatively low-amplitude structures swamped by the low-frequency tail of the dominant peak in the Fourier spectrum are real pulsations. Additional high- $S / N$ photometry may be required to confirm or deny the presence of these periodicities in the lightcurve of PG $1325+101$.

The remaining $12 f_{n}$ frequencies all turn out to be very close to several of the well-secured $F_{n}$ frequencies. This suggests they may be either fine structure, caused by rotational splitting, for instance, or artifacts generated by the prewhitening procedure. Looking at these frequencies in more detail, we note that:

a) $f_{2}$ is approximately $6 \mu \mathrm{Hz}$ away from $F_{3}$. Since the wellidentified triplet $\left(F_{6}, F_{7}, F_{8}\right)$ has a frequency spacing $\Delta v \sim$ $7 \mu \mathrm{Hz}$ (see Paper I and/or the next subsection), it is tempting to interpret $f_{2}$ as a real mode, part of a multiplet split by the star's rotation. This possibility is consistent with our asteroseismic result identifying $F_{3}$ as a nonradial $\ell=1$ mode; 


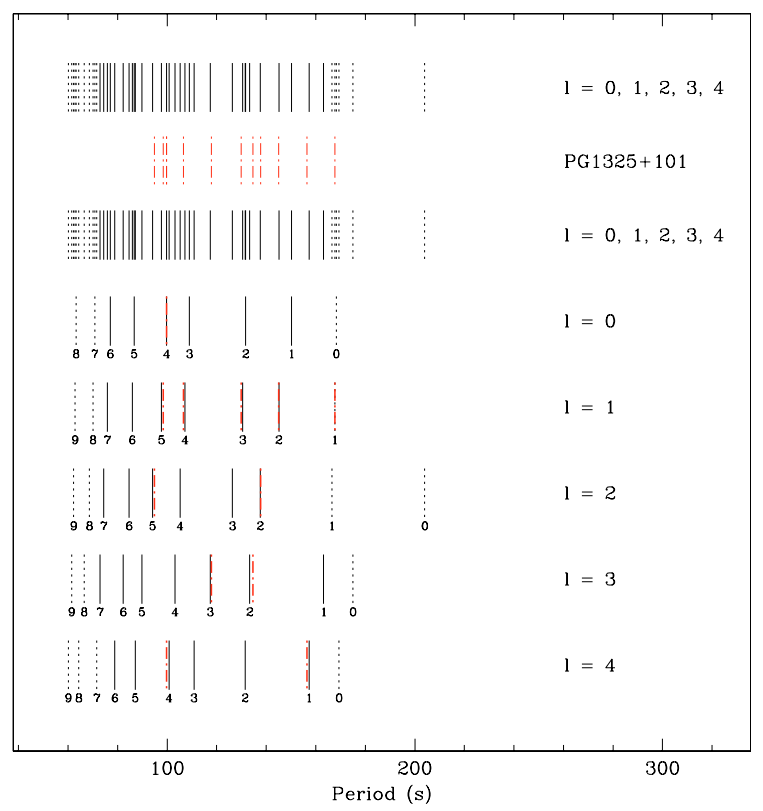

Fig. 6. Comparison of the observed period spectrum of PG 1325+101 (thick dashed-dotted-line red segments) with the theoretical pulsation spectrum of the optimal model. For the latter spectrum, solid-line segments indicate excited modes, while dotted-line segments correspond to damped modes. All pulsation modes with $\ell=0,1,2,3$, and 4 in the period interval $60-210 \mathrm{~s}$ are illustrated. The values of the radial order $k$ are also indicated for each mode. These modes are all acoustic waves (including the $f$-modes). The $g$-modes have periods that fall outside the range of interest for PG $1325+101$.

b) the dominant mode, $F_{4}$, is surrounded by six frequencies $\left(f_{5}-f_{10}\right)$. Among these, $f_{6}$ and $f_{10}$ are separated from $F_{4}$ by $\sim 8 \mu \mathrm{Hz}$ and $\sim 14 \mu \mathrm{Hz}$, respectively. Again, this would be consistent with rotationally split components, this time leading to the interesting constraint that the $F_{4}$ mode must have a degree $\ell=2$ or more (because the frequency separations imply that $\Delta m=2$ between $F_{4}$ and $f_{10}$ and $\Delta m=3$ between $f_{6}$ and $f_{10}$ ). Furthermore, this would support the identification given in Table 2 for that mode. We note, however, that the other frequencies $f_{5}, f_{7}, f_{8}$, and $f_{9}$, if real, do not have the expected spacing for rotationally split components. If $f_{5}$ is far enough away from the other frequencies in this narrow bandpass that it could be considered an independent mode, we find no counterpart in our optimal seismic model that it could be assigned to. This fact along with the very low $S / N$ ratio of that frequency $(\sim 3.8$; see Table 2 of Paper I) may plead for a false detection here. Concerning $f_{9}$, we note that this low-amplitude frequency is approximately $11 \mu \mathrm{Hz}$ away from $F_{4}$, i.e., dangerously close to the 1-day alias of the dominant mode, with a huge amplitude contrast. Hence, the chances are significant that $f_{9}$ may be a spurious residual following prewhitening of the dominant frequency. Finally, both $f_{7}$ and $f_{8}$ are low-amplitude frequencies particularly close to $F_{4}$ (only $3 \mu \mathrm{Hz}$ away) that trigger the same cautionary remarks as above;

c) $f_{11}$ and $f_{12}$ are very close to $F_{5}$ (separated by only $\sim 1 \mu \mathrm{Hz}$ and $\sim 4 \mu \mathrm{Hz}$, respectively). They can hardly be interpreted as multiplet components caused by slow stellar rotation and the mode density they imply, along with their low amplitudes, raise doubts concerning their reality;
Table 3. Inferred structural parameters of PG $1325+101(V=14.019 \pm$ 0.012, Wesemael et al. 1992).

\begin{tabular}{lrlll}
\hline \hline Quantity & \multicolumn{5}{c}{ Estimated value } \\
\hline$T_{\text {eff }}(\mathrm{K})^{\dagger}$ & 35050 & \pm & 220 & $(0.6 \%)$ \\
$\log N(\mathrm{He}) / N(\mathrm{H})^{\dagger}$ & -1.70 & \pm & 0.02 & $(1.2 \%)$ \\
$\log g$ & 5.811 & \pm & 0.004 & $(0.07 \%)$ \\
$M_{*} / M_{\odot}$ & 0.50 & \pm & 0.01 & $(2.0 \%)$ \\
$\log \left(M_{\text {env }} / M_{*}\right)$ & -4.18 & \pm & 0.10 & $(2.4 \%)$ \\
& & & & \\
$R / R_{\odot}\left(M_{*}, g\right)$ & 0.145 & \pm & 0.002 & $(1.5 \%)$ \\
$L / L_{\odot}\left(T_{\text {eff }}, R\right)$ & 28.3 & \pm & 1.5 & $(5.4 \%)$ \\
$M_{\mathrm{V}}\left(g, T_{\text {eff }}, M_{*}\right)$ & 4.45 & \pm & 0.04 & $(0.9 \%)$ \\
$\mathrm{d}\left(V, M_{\mathrm{V}}\right)(\mathrm{pc})$ & 820 & \pm & 21 & $(2.6 \%)$ \\
$P_{\text {rot }}($ day $)$ & 1.6 & \pm & 0.2 & $(12.5 \%)$ \\
$V_{\text {eq }}\left(P_{\text {rot }}, R\right)\left(\mathrm{km} \mathrm{s}^{-1}\right)$ & 4.6 & \pm & 0.6 & $(13.9 \%)$ \\
\hline
\end{tabular}

${ }^{\dagger}$ From spectroscopy.

d) $f_{13}$ and $f_{14}$ are again low-amplitude frequencies, less than $\sim 0.6 \mu \mathrm{Hz}$ away from $F_{7}$ and $F_{8}$, respectively, suggesting that they too are likely artifacts;

e) $f_{15}$ just barely emerges above the noise level $(S / N \sim 3.9)$ and is separated from $F_{11}-F_{12}$ by $10-12 \mu \mathrm{Hz}$, very close to the 1-day alias of these frequencies. The probability of $f_{15}$ being spurious is therefore high.

In summary, among the 15 questionable $\left(f_{n}\right)$ frequencies given in the lower part of Table 2 in Paper I, we find that 4 frequencies $\left(f_{1}, f_{2}, f_{6}, f_{10}\right)$ could indeed easily be interpreted either as independent modes $\left(f_{1}\right)$ or as components of multiplets splitted by the slow rotation of the star $\left(f_{2}, f_{6}, f_{10}\right), 9$ frequencies $\left(f_{5}\right.$, $f_{7}-f_{9}, f_{11}-f_{15}$ ) appear particularly doubtful and could easily be artifacts, and 2 frequencies $\left(f_{3}, f_{4}\right)$ remain ambiguous.

To conclude this subsection, we recall that further checks of the proposed mode identification can possibly be achieved using multicolour photometry. While discriminating between $\ell=0$, 1 , and 2 modes from amplitude ratios at various wavelengths may turn out to be difficult, the signature of the $\ell=3,4$ modes - which are seen in this star according to the fit - should, in principle, be more easily recognized using this technique.

\subsection{Structural parameters of $P G 1325+101$}

The basic properties inferred for PG 1325+101 from the combined spectroscopic and asteroseismic approaches are summarized in Table 3. Following the usual primary quantities ( $T_{\text {eff }}$, $\log g, \log M_{\text {env }} / M_{*} \sim \log q(H)$, and $M_{*}$ ) derived from the parameters of the optimal model, are a set of secondary quantities, functions of the primary values. These are the radius of the star $R$, its luminosity $L$, its absolute magnitude $M_{V}$ (obtained with the use of model atmospheres), and its distance $d$. Estimates of the $1 \sigma$ (internal) errors attached to the primary quantities were derived following Brassard et al. (2001; see also Charpinet et al. 2005b) and are represented in Fig. 3 as red dotted-line boxes. These uncertainties were then used to estimate the errors associated with the secondary parameters.

Additional parameters can be inferred if we interpret the frequency spacing $\Delta v=6.9 \pm 0.7 \mu \mathrm{Hz}$ (mean value; see Paper I) measured for the identified triplet $\left(F_{6}, F_{7}, F_{8}\right)$ as a consequence of the slow rotation of the star. Indeed, the optimal model solution identifies $F_{7}$ as a $\ell=1, k=3$ mode, thus implying that the measured spacing occurs between modes with $\Delta m= \pm 1$. It follows that the rotation period of PG $1325+101$ can be evaluated to $P_{\text {rot }} \simeq\left(1-C_{31}\right) / \Delta v \simeq 1.6 \pm 0.2$ day (using the value 
$C_{31}=0.0140$ given in Table 2). This first-order estimate assumes that the star rotates as a solid body. The rotation period combined with our asteroseismic estimate of the star radius then leads to the evaluation of the equatorial rotation velocity $V_{\text {eq }}=2 \pi R / P_{\text {rot }} \simeq 4.6 \pm 0.6 \mathrm{~km} \mathrm{~s}^{-1}$. Combined with a $V \sin i$ measurement from spectroscopy, it offers, in principle, an opportunity to constrain $i$, the inclination of the rotation axis of the star from the line of sight. Unfortunately, as we saw above, the only limit we got on rotational broadening from our modeling of the He I 4471 line is $V \sin i<20-30 \mathrm{~km} \mathrm{~s}^{-1}$, quite insufficient to constrain the inclination angle since $V_{\mathrm{eq}} \simeq 4.6 \mathrm{~km} \mathrm{~s}^{-1}$. Moreover, such a low equatorial velocity would be hardly measurable based on time-averaged spectra only, as the broadening would be dominated by the pulsations.

Of special interest, we stress that the value derived for the mass of PG $1325+101, M=0.50 \pm 0.01$, coincides with the canonical mass expected from standard evolution and formation scenarios for Extreme Horizontal Branch stars. In addition, the suggestion of a thin H-rich envelope - with $\log \left(M_{\text {env }} / M_{*}\right)=$ $-4.18 \pm 0.10-$ given by this asteroseismic study is consistent with standard expectations for PG $1325+101$, a rather hot and compact EHB star.

\section{Summary and conclusion}

Following the results, reported in Paper I, of a multisite campaign of time-series photometry dedicated to the relatively bright $(V=14.019 \pm 0.012)$, rapidly pulsating hot B subdwarf PG $1325+101$, we conducted a thorough analysis of the properties of this star using new, improved spectroscopic evaluations of its atmospheric parameters to perform a detailed asteroseismic study of its pulsations.

Our approach relied on the "forward modeling" method with the goal of objectively finding the model that would best match the 12 periods identified as independent oscillation modes in PG $1325+101$ with a set of theoretical periods. For this purpose, we have used the second generation sdB models of Charpinet et al. (1997) to compute the theoretical periods, since these have proved to account quite well for the class properties of the EC14026 pulsators (Charpinet et al. 2001). Our exhaustive exploration of the vast model parameter space, combined with the spectroscopic constraints, then allowed us to isolate a unique seismic model solution that best reproduces the observed periods of PG $1325+101$. From this model we derived the main structural properties of this star as well as the identification of the modes responsible for the luminosity variations.

The basic properties of PG 1325+101 inferred from our combined spectroscopic and asteroseismic approach are summarised in Table 3. Once the appropriate seismic solution has been identified with the help of spectroscopy, fundamental parameters, such as the surface gravity $\log g$, could be determined with a much higher precision. In addition, measurements of the H-rich envelope mass (a pure product of asteroseismology) and the total mass of the star could be derived. These quantities, in particular, will be of prime importance to constrain scenarios of the evolution and formation of Extreme Horizontal Branch stars and their progenitors. In our study of PG $1325+101$, we found that the total mass could not be inferred independently of the spectroscopic measurement of the effective temperature. Nonetheless, the value derived for the total mass, $M=0.50 \pm 0.01 M_{\odot}$, indicates a mass of PG $1325+101$ that is close to the value commonly admitted for sdB stars. Although this value depends on the reliability of the spectroscopic estimate of $T_{\text {eff }}$ (particularly regarding eventual systematic effects), we stress that masses significantly different from the derived, nearly canonical value, such as some scenarios of binary evolution suggest, would require a shift of several thousand Kelvins in effective temperature to be compatible with asteroseismology. Such a drastic change in the evaluation of $T_{\text {eff }}$ from spectroscopy is highly unlikely and, therefore, we are confident in concluding that PG 1325+101 has a mass close to the canonical mass of extreme horizontal branch stars. Interestingly, we point out that current evidence strongly suggests an absence of orbital motion due to the presence of a close companion (e.g., Telting \& Østensen 2004), although it cannot be completely ruled out at the present time, for instance, if the inclination is large and the orbital period relatively long (several days). If PG $1325+101$ is confirmed as a single sdB star with the mass given from asteroseismology, it would add to the growing constraint already posed by other similar single sdB pulsators regarding the hypothesis that mergers of two helium white dwarfs may be the progenitors of such stars. Notably, we also found that PG $1325+101$ has a thin hydrogen-rich envelope mass - with $\log \left(M_{\text {env }} / M_{*}\right)=-4.18 \pm 0.10-$ as one would expect for a rather hot and compact EHB star (Dorman et al. 1993). Also of interest, we found asteroseismic suggestions that PG $1325+101$ is a slow rotator, with a rotation period of $P_{\text {rot }}=1.6 \pm 0.2$ days, consistent with the independent, albeit loose, spectroscopic constraint of $V \sin i<20-30 \mathrm{~km} \mathrm{~s}^{-1}$.

Finally, we found that our optimal model solution is able to reproduce simultaneously the 12 independent periods observed in PG $1325+101$ with an average dispersion of only $\sim 0.46 \%$ (or $\sim 0.54 \mathrm{~s}$, on an absolute scale). The observed periods correspond to low-order $\ell=0-4$ modes which, indeed, are expected to be observable according to nonadiabatic pulsation theory. Hence, PG 1325+101 constitutes another case for which a seismic model solution exists and can, at the same time, reproduce all the periods observed in this star at a high level of accuracy, be consistent with nonadiabatic theory, and satisfy the spectroscopic constraints. Such a consistency between three independent aspects of the modeling of these pulsating stars is a result that was not guaranteed at the outset. This is a strong indication that the basic constitutive physics incorporated into our current models used for the asteroseismic analyses of EC14026 stars is sound, especially as it follows similar results already obtained for the rapid sdB pulsators PG 0014+067, PG 1047+003, PG 1219+534, and Feige 48 (Brassard et al. 2001; Charpinet et al. 2003, 2005b,a). Furthermore, the excellent agreement between observations and theory at the nonadiabatic level adds another confirmation that the iron bump opacity mechanism of Charpinet et al. (1997) is at the origin of the EC14026 phenomenon.

Acknowledgements. This work was supported in part by the NSERC of Canada and by the Fund FQRNT (Québec). G.F. also acknowledges the contribution of the Canada Research Chair Program. T.D.O. acknowledges support from NSF AST-0206115.

\section{References}

Brassard, P., Fontaine, G., Billères, M., et al. 2001, ApJ, 563, 1013 Brassard, P., Pelletier, C., Fontaine, G., \& Wesemael, F. 1992, ApJS, 80, 725 Brown, T. M., Ferguson, H. C., Davidsen, A. F., \& Dorman, B. 1997, ApJ, 482, 685

Charpinet, S., Fontaine, G., Brassard, P., et al. 1997, ApJ, 483, L123 Charpinet, S., Fontaine, G., \& Brassard, P. 2001, PASP, 113, 775 Charpinet, S., Fontaine, G., \& Brassard, P. 2003, in White Dwarfs, NATO ASIB Proc., 105, 69

Charpinet, S., Fontaine, G., Brassard, P., et al. 2005a, A\&A, 443, 251 Charpinet, S., Fontaine, G., Brassard, P., Green, E. M., \& Chayer, P. 2005b, A\&A, 437, 575 
Chayer, P., Green, E. M., Fontaine, G., \& Saffer, R. A. 2003, in White Dwarfs, ed. D. de Martino, R. Silvotti, J.-E. Solheim, \& R. Kalytis (Dordretch: Kluwer), NATO Science Series, 105,85

Dorman, B., Rood, R. T., \& O'Connell, R. W. 1993, ApJ, 419, 596

Fontaine, G., \& Brassard, P. 1994, in Stellar and Circumstellar Astrophysics, a 70th birthday celebration for K. H. Bohm \& E. Bohm-Vitense, ASP Conf. Ser., 57, 195

Fontaine, G., Brassard, P., Charpinet, S., et al. 2003, ApJ, 597, 518

Fontaine, G., Brassard, P., Charpinet, S., \& Chayer, P. 2006, in Stellar Pulsation and Evolution, Mem. S. A. It., 77, 49

Green, E. M., Fontaine, G., Reed, M. D., et al. 2003, ApJ, 583, L31

Han, Z., Podsiadlowski, P., Maxted, P. F. L., \& Marsh, T. R. 2003, MNRAS, 341 669

Heber, U., Reid, I. N., \& Werner, K. 2000, A\&A, 363, 198

Heber, U. 1986, A\&A, 155, 33

Hubeny, I., \& Lanz, T. 1995, ApJ, 439, 875

Jeffery, C. S., Dhillon, V., Marsh, T., \& Ramachandran, B. 2004, MNRAS, 352, 699

Jeffery, C. S., Aerts, C., Dhillon, V. S., Marsh, T. R., \& Gänsicke, B. T. 2005, MNRAS, 362, 66

Kawaler, S. D., \& Hostler, S. R. 2005, ApJ, 621, 432

Kilkenny, D., Koen, C., O’Donoghue, D., \& Stobie, R. S. 1997, MNRAS, 287, 867

Lanz, T., \& Hubeny, I. 1995, ApJ, 439, 905

O’Donoghue, D., Koen, C., Lynas-Gray, A.E., Kilkenny, D., \& van Wyk, F. 1998, MNRAS, 296, 306

Randall, S. K., Fontaine, G., Brassard, P., \& Bergeron, P. 2005, ApJS, 161, 456

Randall, S. K., Fontaine, G., Green, E. M., et al. 2006, ApJ, 643, 1198

Saffer, R. A., Bergeron, P., Koester, D., \& Liebert, J. 1994, ApJ, 432, 351

Silvotti, R., Østensen, R., Heber, U., et al. 2002, A\&A, 383, 239

Silvotti, R., Bonanno, A., Bernabei, S., et al. 2006, A\&A, 459, 557 (Paper I),

Solheim, J.-E., Østensen, R., Silvotti, R., \& Heber, U. 2004, Ap\&SS, 291, 419

Telting, J. H., \& Østensen, R. H. 2004, A\&A, 419, 685

Wesemael, F., Fontaine, G., Bergeron, P., Lamontagne, R., \& Green, R. F. 1992, AJ, 104, 203

1 UMR 5572, Université Paul Sabatier et CNRS, Observatoire MidiPyrénées, 14 Av. E. Belin, 31400 Toulouse, France e-mail: scharpin@ast.obs-mip.fr

2 INAF - Osservatorio Astronomico di Capodimonte, via Moiariello 16, 80131 Napoli, Italy

e-mail: silvotti@na.astro.it
3 INAF - Osservatorio Astronomico di Catania, via S. Sofia 78, 95123 Catania, Italy e-mail: [alfio;leccia;afr]@ct.astro.it

4 Département de Physique, Université de Montréal, CP 6128, Succursale Centre-Ville, Montréal, QC, H3C 3J7, Canada e-mail: [fontaine; brassard; bergeron] @astro . umontreal . ca

5 Dept. of Physics and Astronomy, Johns-Hopkins University, 3400 North Charles St., Baltimore, MD 21218-2686, USA e-mail: chayer@pha.jhu.edu

6 Primary affiliation: Dept. of Physics and Astronomy, University of Victoria, PO Box 3055, Victoria, BC V8W 3P6, Canada

7 Steward Observatory, University of Arizona, 933 North Cherry Av., Tucson, AZ 85721, USA e-mail: bgreen@as . arizona.edu

8 INAF - Osservatorio Astronomico di Bologna, via Ranzani 1, 40127 Bologna, Italy

e-mail: bernabei@astbo3.bo.astro.it

9 Danish Asteroseismology Center, Institut for Fysik og Astronomi, Aarhus Universitet, Ny Munkegade, Bygn. 1520, 8000 Aarhus C., Denmark e-mail: kjeldsen@phys .au.dk

10 Institute of Theoretical Physics and Astronomy, Vilnius University, Lithuania e-mail: jr@itpa.1t

11 Isaac Newton Group, S. Cruz de la Palma, Canary Islands, Spain e-mail: roy@ing.iac.es

12 Korea Astronomy and Space Science Institute, South Korea e-mail: [slkim; bgpark] @kasi .re.kr

13 National Astronomical Observatories, Beijing, 100012, PR China e-mail: xjjiang@bao.ac.cn

14 Missouri State University and Baker Observatory, 901 S. National, Springfield, MO 65897, USA e-mail: [MikeReed; gww836f;rsp814f] @missouristate.edu

15 Wise Observatory, Israel e-mail: [yiftah;lili;elia]@wise.tau.ac.il

16 Florida Institute of Technology, USA e-mail: [toswalt; rudkin;kyjohnst] afit.edu 